\title{
Breast MRI Tumour Segmentation using Modified Automatic Seeded Region Growing Based on Particle Swarm Optimization Image Clustering
}

\author{
Ali Qusay Al-Faris ${ }^{1}$, Umi Kalthum Ngah $^{1}$, Nor Ashidi Mat Isa ${ }^{1}$, Ibrahim Lutfi \\ Shuaib $^{2}$ \\ ${ }^{1}$ Imaging and Computational Intelligence Research Group (ICI), Universiti Sains Malaysia, \\ Penang, Malaysia \\ Email:alialfaris2009@gmail.com, \\ eeumi@eng.usm.my, ashidi@eng.usm.my \\ ${ }^{2}$ Advanced Medical and Dental Institute (AMDI), Universiti Sains Malaysia, Penang, Malaysia \\ E-mail: ibrahimeamdi.usm.edu.my
}

\begin{abstract}
In this paper, a segmentation system with a modified automatic Seeded Region Growing (SRG) based on Particle Swarm Optimization (PSO) image clustering will be presented. The paper is focused on Magnetic Resonance Imaging (MRI) breast tumour segmentation. The PSO clusters' intensities are involved in the proposed algorithms of the automated SRG initial seed and threshold value selection. Prior to that, some pre-processing methodologies are involved. And breast skin is detected and deleted using the integration of two algorithms, i.e. Level Set Active Contour and Morphological Thinning. The system is applied and tested on the RIDER breast MRI dataset, and the results are evaluated and presented in comparison to the Ground Truths of the dataset. The results show higher performance compared to the previous segmentation approaches that have been tested on the same dataset.
\end{abstract}

Keywords: Breast MRI, PSO Image Clustering, Tumour Segmentation, Seeded Region Growing, Level Set Active Contour, Morphological Thinning.

\section{Introduction}

Breast cancer today is the leading cause of death amongst cancer patients inflicting women around the world. To date, 1.38 million new breast cancer cases have been diagnosed, which is $23 \%$ of total new cancer cases in the world. 458,400 breast cancer death cases have been recorded making up $14 \%$ of the total cancer deaths in 2008 alone [1]. Several of the commonly used medical screening techniques used for breast screening are mammography, ultrasound and MRI. While the produced images by mammogram demonstrates the contrast between soft tissue and bone density, MRI on

adfa, p. 1, 2011.

(C) Springer-Verlag Berlin Heidelberg 2011 
the other hand, produces clear and crisp images, which provides a better contrast between different kinds of soft tissue. For that reason, MRI is used for breast screening i.e. to explore the small details between breast tissues. Although this is valuable information, the presented data still needs to be interpreted by the radiologist [2]. For this purpose, image processing methods are used to assist the radiologists in improving the quality of these medical images and in detecting tumour masses.

\section{Related Work}

Several techniques have been developed and also evolved for medical image segmentation. These includes methods such as Particle Swarm Optimization (PSO) [3], Genetic Algorithm [4] and Artificial Fuzzy Logic [5-7]. The supervised, unsupervised and semi-supervised methods are explored in Azmi et al's study [8]. In their comparison study on MRI Breast RIDER dataset, the researchers found that the supervised segmentation methods such as; K-Nearest Neighbors (KNN), Support Vector Machine (SVM) and Bayesian and the semi-supervised method such as self training and improved self-training (IMPST) lead to high accuracy. However labeled data is needed. Hence, the process becomes difficult, expensive, and involves a lot of time. On the other hand, unsupervised methods such as; Fuzzy C-means (FCM) need no prior knowledge. However, the performance is low [8]. The Seeded Region Growing algorithm which was proposed by Adams and Bischof [9] is widely used in the medical images today because it effectively segments different types of images[10]. In Meinel's study on MRI breast segmentation [11] the SRG was also used. The experiments on breast tumour segmentation returned robust results. However, this approach needed an initial threshold value to be specified by the user. This is used to find the anticipated locations of the tumors. The SRG Feature Extraction algorithm has been proposed on cervical cancer screening. This algorithm extracted four cervical cells features; size of nucleus, cytoplasm, grey level of nucleus and cytoplasm. The data extracted using SRGFE algorithm gave high correlation value when compared with data extracted manually. Still, the user needs to determine the region of interest to select the initial seed pixel. The user also needs to determine the threshold value [12]. $\mathrm{Wu}$ et al. proposed texture feature-based automated SRG algorithm on abdominal organ segmentation [13]. The advantage of this algorithm is that it allows minimum user intervention. This is helpful for batch work. However, this approach does have drawbacks. Texture feature based methods all have the assumption that the region should have texture homogeneity. For organs with complex texture, this approach may not work well. Shan et al. proposed an automatic seed point selection algorithm for SRG on ultrasound breast images [14]. The algorithm needs no prior information or training process. Both the homogeneous texture features and spatial features of the breast tumors are taken into account. However, some cases failed because of the shadowing effects of areas having similar intensity as the tumor and right below the tumor. In this study, a proposed segmentation approach with automated features for MRI breast tumor segmentation is presented using modified automatic SRG method based on PSO image clustering. 


\section{Proposed Approach}

The proposed approach starts with a pre-processing phase which is followed by the detection and deletion of the breast skin. Then automatic seed and threshold selection processes are presented based on PSO image clustering before the SRG algorithm is applied. The whole perspective is shown as in Fig. 1.

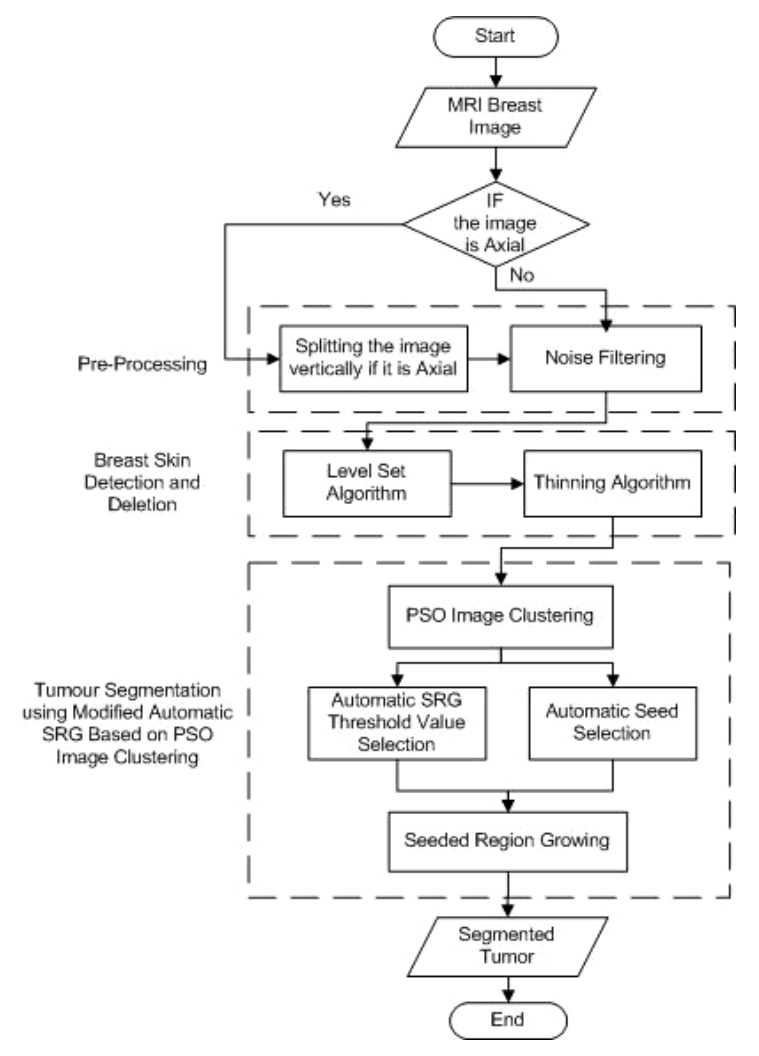

Fig. 1. Methodology Flowchart

\subsection{Image Pre-Processing}

The pre-processing phase is the first process that is executed. The image is split into two sub-images; the right breast image and the left breast image. This process is used only if the MRI breast image is Axial (i.e. the image is taken from the perspective of the patient from head to toe). This process is skipped if the image is Sagittal (i.e. the image is taken according to the lateral view). The splitting process can be done by finding the middle of the X-coordinate of the image and splitting the image vertically from that point. The median filter is then applied in order to enhance the images' resolution and to reduce the presence of the salt and pepper noise while the boundaries and features are kept intact [15]. 


\subsection{Breast Skin Detection and Deletion}

The purpose of this process is to delete the breast skin area which has similar intensity range compared to the tumor area's intensity range. This process is also necessary in order to facilitate a better automatic seed selection for the tumor segmentation in the next stage. To delete the breast skin, an integration of Level Set Active Contour algorithm [16] with Morphological Thinning Algorithm is used in this phase. The Level Set Active Contour (Chunming's algorithm) is used to detect the breast skin border; the algorithm is dynamic curves that move toward the mass border. An external energy moves the zero level curves toward the mass border using the edge indicator function $g$ that is defined in (1)

$$
g=\frac{1}{1+\left|\nabla G_{\sigma} * I\right|^{2}}
$$

where $I$ is the image, $G_{\sigma}$ is the Gaussian kernel with a standard deviation $\sigma$. By changing the $\sigma$ parameter value and the number of the iterations, In order to delete the detected breast skin border, the Morphological Thinning Algorithm [17] is used. The thinning level depends on the number of iterations. Whenever the number of iterations is increased, there would be more shrinking of the image's border. The Morphological Thinning Algorithm only accepts a binary version of the image. Therefore, the resultant image after the Chunming's algorithm would be converted to binary image. Furthermore, after applying the thinning, the binary image is reconverted into its original grey scale representation.

\subsection{A Modified Automatic SRG Based On PSO Image Clustering}

In this study, the SRG algorithm for tumour segmentation is chosen because it is fast, simple and robust[18]. While the chosen image clustering method is PSO-based, because it produces better results compared with other clustering methods such as $\mathrm{K}$ means, Fuzzy C-means, K-Harmonic means and Genetic Algorithms[19-21].

\section{Seeded Region Growing (SRG).}

SRG [9] starts with an initial seed pixel and tries to compare their neighborhood pixels with the seed according to some attributes, such as the intensity or texture. It then merges them if they are similar enough. The eight neighbor pixels will be tested according to the intensity next sorted into the segmented pixel list if the tested neighbor pixel has similar intensity. Subsequently, the eight neighbors of the new pixel will be tested and sorted too. The process then continues in the same manner. Fig. 2 shows the initial seed pixel and the eight neighbors. SRG has two variable factors which are usually selected manually. The first factor is determining the initial seed pixel that the SRG can start growing. The second factor is the threshold value for measuring the difference between the pixel and their neighbors. In this work, an automated version of the seed selection algorithm and SRG threshold based on the PSO image clustering are presented.

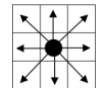

Fig. 2. The initial seed pixel and their eight neighbor pixels 


\section{Particle Swarm Optimization (PSO) Image Clustering.}

Applying the PSO image clustering would be organizing the image into groups whose members are having similar intensity range. Therefore, each cluster represents different intensity range of image. Various versions of image clustering based on PSO have been proposed in[19-21]. The method used in this paper proposed in [19] and described in [21] as below:

$N_{c}$ is the number of clusters to be formed, $Z_{p}$ is the p-th pixel, $C_{j}$ is the subset of pixel vectors that form cluster $\mathrm{j}, x_{i}=\left(m_{i 1}, \ldots m_{i j}, \ldots m_{i N_{c}}\right)$ where $m_{i j}$ refers to the $\mathrm{j}$ th cluster centroid vector of the i-th particle.

1. Initialize each particle to contain $N_{c}$ randomly selected cluster means.

2. FOR $\mathrm{t}=1$ to $t_{\max }$ (maximum number of iterations)

FOR each particle i

FOR each pixel $Z_{p}$

Calculate $d\left(Z_{p}, m_{i j}\right)$ for all clusters $C_{i j}$

Assign $Z_{p}$ to $C_{i j}$ where

$$
d\left(Z_{p}, m_{i j}\right)=\min _{\forall c=1, \ldots, N_{c}}\left\{d\left(Z_{p}, m_{i c}\right)\right\}
$$

$d\left(Z_{p}, m_{i j}\right)$ represents the Euclidean distance between the p-th pixel zp and the centroid of $\mathrm{j}$-th cluster of particle $\mathrm{i}$.

Calculate the fitness function $f\left(x_{i}(t), Z\right)$ where $\mathrm{Z}$ is a matrix representing the assignment of pixels to clusters of particle i.

Update the personal best and the global best positions.

Update the cluster centroids using equations (2) and (3).

$v_{i}(t+1)=w v_{i}(t)+c_{1} r_{1}(t)\left(y_{i}(t)-x_{i}(t)\right)+c_{2} r_{2}(t)\left(y_{g}(t)-x_{i}(t)\right)$

$x_{i}(t+1)=x_{i}(t)+v_{i}(t+1) \quad(3)$

Where $x_{i}$ is the current position of the particle, $v_{i}$ is the current velocity of the particle, $y_{i}$, is the best position that particle has achieved so far, $y_{g}$ is the location of overall best value, $w$ is the inertia weight, $c_{1}$ and $c_{2}$ are the acceleration constants, and $r_{1}(t)$ and $r_{2}(t)$ are random numbers generated in the range between 0 and 1 .

3. Segment the image using the optimal number of clusters and the optimal clusters centroids given by the best global particle.

\section{The Proposed Automatic SRG Initial Seed Selection.}

After applying the PSO image clustering, the clusters' intensities would be ranked in ascending order. Subsequently, the cluster which has the highest intensity would be chosen. Then the centre of the chosen cluster region is selected as the initial seed. The steps taken for this process are as follows:

1. Apply PSO Image clustering on the MRI breast image.

2. Rank the PSO clusters according to their intensity values in ascending order.

3. Select the regions with the highest clusters' intensity values and eliminate the other cluster regions.

4. Find the position ( $x, y$ coordinates) of the center pixel of the maximum area in the selected regions.

5. Set the selected position in step 4 as the position of the initial seed. 
The Proposed Automatic SRG Threshold Value Selection.

The importance of this process is because of the ranges of the grayscale representations for the tumour and the other parts of the breast are not consistent from one image to another. Therefore, the proposed method has the capability of changing the SRG threshold value according to the respective image's gray scale distribution. The method is based upon finding the optimum estimated value from the PSO clusters' intensities mean values. The average for clusters' intensities except the highest cluster's intensity (which contains the tumor region) has to be calculated first using equation (4).

$$
\text { CAvg }=\frac{\sum_{i=0}^{N_{c}-1} C I_{i}}{N_{c}-1}
$$

Where $i$ is the PSO clusters counter, $N_{c}$ is the maximum clusters number, $C I$ is the cluster intensity and CAvg is the clusters' intensity average.

The CAvg is used to examine the optimum SRG threshold value as described in the pseudo code:

SET Zero to (IntensitySum), Set DefaultValue (the default value is value between CAvg-255)

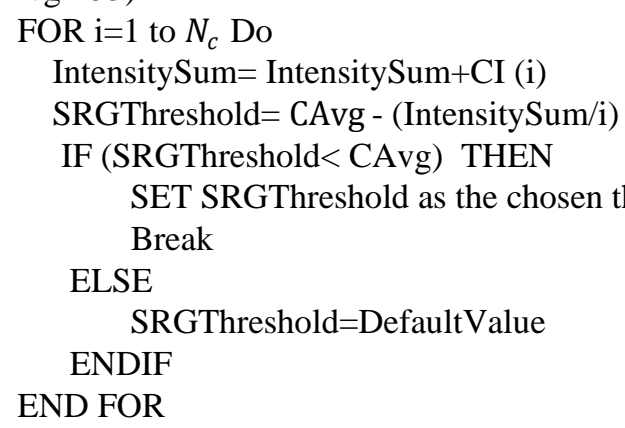

\section{Experimental Results and Discussion}

The methodology explained earlier is applied and tested on the RIDER Breast MRI dataset which is downloaded from the National Biomedical Imaging Archive (NBIA)[22]. This website belongs to the U.S. National Cancer Institute. The dataset includes breast MRI images for five patients. All images are Axial 288 X 288 pixels. The dataset also include Ground Truth (GT) segmentation, which have been identified manually by a radiologist. Three sequences with their GT are selected for each patient to be used in the experiments as test images. GT is used as a benchmark for performance evaluation of segmentation methods in our experiments. Fig 3 illustrates one RIDER MRI breast image after applying the different processes of the approach. 


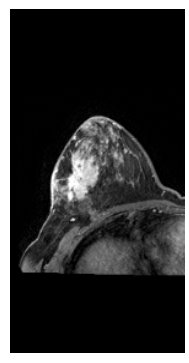

(a)

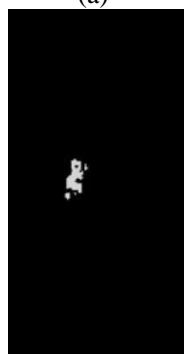

(e)

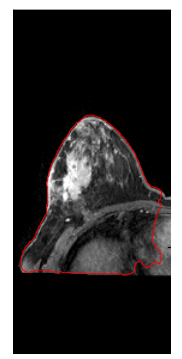

(b)

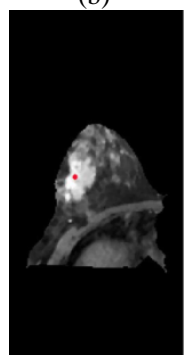

(f)

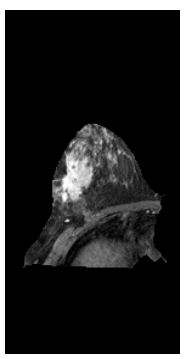

(c)

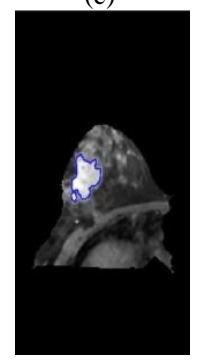

(g)

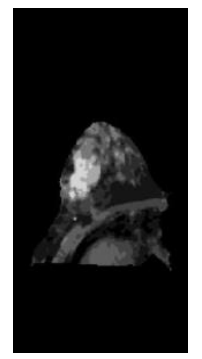

(d)

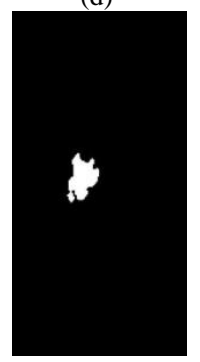

(h)

Fig. 3. The tumor segmentation process applied on RIDER MRI image (a) MRI image after splitting process. (b) Breast skin detected by the Level Set Algorithm. (c) Breast skin deleted by the Thinning Algorithm. (d) The resultant images after applying the PSO image clustering. (e) The highest PSO clusters' intensity region after other regions are eliminated. (f) Initial seed selected automatically (marked in red) as the center of the region selected in (e). (g) SRG using the automatic threshold value is applied (marked in blue). (h) The segmented tumor area.

Different measures are used in this study in order to evaluate the segmentation accuracy. The number of pixels of $\left(R_{s}\right)$ and $\left(R_{t}\right)$ have to be found first, where $\left(R_{s}\right)$ represents the segmented region by the proposed approach, while $\left(R_{t}\right)$ represents the ground truth regions segmented by the experts.

The evaluation measures used in this study are; True Positive Fraction (TPF) (also called Sensitivity), True Negative Fraction (TNF) [23-26], Relative Overlap (RO) (also called segmentation precision) and Misclassification Rate (MCR) which have been used before for brain segmentation [27] and in breast segmentation [8, 28]. The calculations are made using the equations 5-8.

$$
\begin{aligned}
& T P F=\frac{R_{s} \cap R_{t}}{R_{t}} \\
& T N F=1-\frac{R_{s}-R_{t}}{R_{t}} \\
& R O=\frac{R_{s} \cap R_{t}}{R_{s} \cup R_{t}} \\
& M C R=1-\frac{R_{s} \cap R_{t}}{R_{t}}
\end{aligned}
$$

The results of the proposed approach are compared with the results of the previous works involving five different segmentation approaches. The previous work's results have been stated in the comparison study of [8]. The approaches are K-Nearest Neighbors (KNN), Support Vector Machine (SVM), Bayesian, Fuzzy C-means (FCM) and Improved Self-Training (IMPST). The tested data are the same dataset 
which is (Breast MRI RIDER dataset). The results of the five approaches and the proposed approach are stated in Table 1.

Table 1. Segmentation results for the proposed approach and other approaches (K.N.N, SVM, Bayesian, FCM and IMPST).

\begin{tabular}{|c|c|c|c|c|c|c|c|c|c|c|c|c|}
\hline $\begin{array}{c}\text { The } \\
\text { method }\end{array}$ & \multicolumn{2}{|c|}{ KNN } & \multicolumn{2}{|c|}{ SVM } & \multicolumn{2}{|c|}{ Bayesian } & \multicolumn{2}{|c|}{ FCM } & \multicolumn{2}{|c|}{ IMPST } & \multicolumn{2}{|c|}{$\begin{array}{l}\text { The Proposed } \\
\text { Approach }\end{array}$} \\
\hline Statistic & Mean & Stdev & Mean & Stdev & Mean & Stdev & Mean & Stdev & Mean & Stdev & Mean & Stdev \\
\hline RO & 595 & 121 & 0.6 & 187 & 555 & 174 & 0.587 & 0.250 & 0.677 & 0.167 & 0.704 & 0.143 \\
\hline MSC & 0.27 & 0.87 & 0.25 & 0.81 & 0.24 & 0.81 & 0.18 & 0.73 & 0.21 & 0.81 & 0.209 & 0.133 \\
\hline TPF & 0.73 & 0.13 & 0.75 & 0.19 & 0.76 & 0.19 & 0.82 & 0.27 & 0.79 & 0.19 & 0.792 & 0.133 \\
\hline TNF & 0.75 & 0.15 & 0.71 & 0.21 & 0.59 & 0.25 & 0.42 & 0.55 & 0.83 & 0.15 & 0.851 & 0.237 \\
\hline
\end{tabular}

The results of the proposed approach show improved performance compared with the results of the previous approaches. The proposed approach not only provides improved performance, it also facilitates automated selection of the suspected regions without the need to manually select the region of interest (ROI) as is necessary in the previous approaches. Table 1 show the results of the RO mean (0.704) and TNF mean (0.851) of the proposed approach are improved compared with the previous approaches. Nevertheless, the TPF mean (0.792) and MCS mean (0.209) of the proposed approach are superior to the K.N.N, SVM, Bayesian and IMPST approaches. However, the values are less in accuracy when compared with one approach (the FCM) because it uses manually drawn ROI to select the suspected window before starting the segmentation process. Fig. 3 shows the Receiver Operating Characteristic (ROC) curve which is used to illustrate the True Positive Fraction compared with the False Positive Fraction.

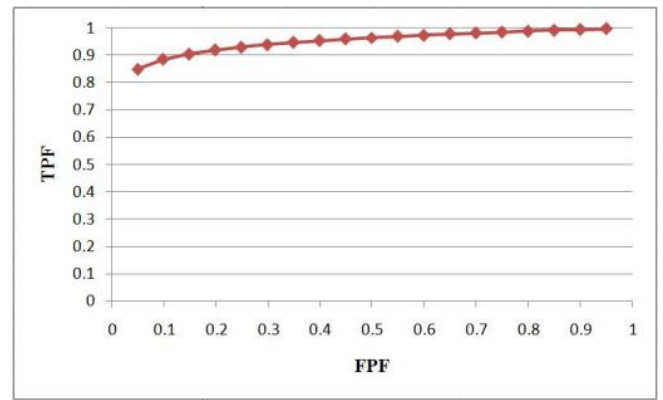

Fig. 4. The ROC curve for all tested RIDER dataset images.

From the curve, it can be observed that the Area under the Curve (AUC) is 0.95. The high AUC indicates improved segmentation performance. 


\section{Conclusion}

A modified automatic Seeded Region Growing based on PSO image clustering system for MRI breast tumour segmentation has been presented in this paper. The modification has been made by proposing two automatic approaches for selecting the SRG variable factors which are usually selected manually. The first approach selects the position of the initial seed pixel; along with the second approach which determines the SRG threshold value for measuring the difference between the pixel and their neighbors. Both approaches are based on the clusters' intensities of the PSO image clustering. Prior to that, some necessary pre-processing processes are made such as; splitting the axial images, noise reduction and deletion of the breast skin using the integration of Level Set Active Contour and Morphological Thinning algorithms. The study is then supported by the results of applying the methodology on the RIDER breast MRI dataset. The evaluation has been made using the ground truth of the dataset as a benchmark. The results are then compared with previous works, which are also based on the same dataset. Not only is the performance significantly improved; the proposed approach also avoided the need for manual selection of the suspected region window, seed pixel and threshold value processes. These processes are replaced with automated methods. The methods are also generic for any grayscale representation of the breast MRI images.

\section{References}

1. Jemal, A., et al., Global Cancer Statistics. CA: A Cancer Journal for Clinicians, 2011. 61(2): p. 69-90.

2. Gardiner, I., CAD Improves Breast MRI Workflow: Increasing Throughput While Maintaining Accuracy in Breast MRI Reads Requires Powerful Workflow Tools, in Imaging Technology News2010.

3. Ibrahim, S., N.E.A. Khalid, and M. Manaf, Empirical Study of Brain Segmentation using Particle Swarm Optimization, in International Conference on Information Retrieval and Knowledge Management, CAMP102010: Shah Alam, Selangor. p. 235-239.

4. Ganesan, R. and S. Radhakrishnan, Segmentation of Computed Tomography Brain Images using Genetic Algorithm. International Journal of Soft Computing, 2009. 4(4): p. 157-161.

5. Hussain, R., et al., Fuzzy Clustering Based Malignant Areas Detection In Noisy Breast Magnetic Resonant (MR) Images. International Journal of Academic Research, 2011. 3(2).

6. Kannan, S., A. Sathya, and S. Ramathilagam, Effective Fuzzy Clustering Techniques For Segmentation of Breast MRI. Soft Computing - A Fusion of Foundations, Methodologies and Applications, 2011. 15(3): p. 483-491.

7. Noor, N.M., et al., Adaptive Neuro-Fuzzy Inference System for Brain Abnormality Segmentation, in 2010 IEEE Control and System Graduate Research Colloquium, ICSGRC 2010.2010.

8. Azmi, R., et al., IMPST: A New Interactive Self-Training Approach to Segmentation Suspicious Lesions in Breast MRI. J Med Signals Sens, 2011. 1(2): p. 138-48.

9. Adams, R. and L. Bischof, Seeded Region Growing. IEEE Trans. Pattern Anal. Machine Intell, 1994. 16: p. 641-647. 
10. Khalid, N.E.A., et al., Seed-Based Region Growing Study for Brain Abnormalities Segmentation, in International Symposium on Information Technology 2010 (ITSim 2010)2010: Kuala Lumpur. p. 856-860

11. Meinel, L.A., Development of computer-aided diagnostic system for breast MRI lesion classification." dissertation, , in Biomedical Engineering2005, University of Iowa: Iowa.

12. Mat-Isa, N.A., M.Y. Mashor, and N.H. Othman, Seeded Region Growing Features Extraction Algorithm; Its Potential Use in Improving Screening for Cervical Cancer. International Journal of The Computer, The Internet and Management, 2005. 13(1).

13. Wu, J., et al. Texture Feature based Automated Seeded Region Growing in Abdominal MRI Segmentation. in BioMedical Engineering and Informatics. BMEI 2008. 2008. Sanya.

14. Shan, J., H.D. Cheng, and Y. Wang. A Novel Automatic Seed Point Selection Algorithm For Breast Ultrasound Images. in Pattern Recognition, 2008. ICPR 2008. 19th International Conference. 2008.

15. Chun-yu, N., L. Shu-fen, and Q. Ming, Research on Removing Noise in Medical Image Based on Median Filter Method, in IT in Medicine \& Education, 2009. ITIME '09. IEEE International Symposium, I.C. Publications, Editor 2009. p. 384 - 388.

16. Li, C., et al. Level Set Evolution Without Re-initialization: A New Variational Formulation. in IEEE International Conference on Computer Vision and Pattern Recognition (CVPR). 2005. San Diego.

17. Lam, L., Seong-Whan Lee, and C.Y. Suen, Thinning Methodologies-A Comprehensive Survey. IEEE Transactions on Pattern Analysis and Machine Intelligence, 1992. 14(9): p. 879.

18. Ibrahim, S., et al., Particle Swarm Optimization vs Seed-Based Region Growing: Brain Abnormalities Segmentation. International Journal of Artificial Intelligence, 2011. 7(1): p. 174-188.

19. Omran, M.G.H., A PSO-based Clustering Algorithm with Application to Unsupervised Image Classification, 2005, University of Pretoria etd.

20. Ouadfel, S., M. Batouche, and A. Taleb-Ahmed. A Modified Particle Swarm Optimization Algorithm for Automatic Image Clustering. in International Symposium on Modelling and Implementation of Complex Systems, MISC'2010. 2010.

21. Wong, M.T., X. He, and W.-C. Yeh, Image Clustering Using Particle Swarm Optimization. 2011.

22. "RIDER Breast MRI", National Biomedical Imaging Archive (NBIA), U.o. Michigan, Editor 2007, U.S. National Cancer Institute.

23. Chalana, V. and Y. Kim, A Methodology For Evaluation of Boundary Detection Algorithms on Medical Images. IEEE Transactions on Medical Imaging, 1997. 16: p. 642-652.

24. Fenster, A. and B. Chiu. Evaluation of Segmentation algorithms for Medical Imaging. in IEEE Engineering in Medicine and Biology 27th Annual Conference. 2005. Shanghai, China.

25. Metz, C.E., ROC methodology in radiologic imaging. Investigative Radiology, 1986. 21: p. 720-733.

26. McNeil, B.J. and J.A. Hanley, Statistical approaches to analysis of receiver operating characteristic ROC curves. Medical Decision Making, 1984. 14: p. 137-150.

27. T. Song, et al., A hybrid tissue segmentation approach for brain MR images. Med. Biol. Eng. Comput., 2006. 44: p. 242. 
28. Gökhan Ertas, et al., Breast MR segmentation and lesion detection with cellular neural networks and 3D template matching. Computers in Biology and Medicine, 2008. 38: p. 116 $-126$. 